\title{
Particle Size Determination in SBA15 Nanocomposite Using Model Based Analysis of SANS and Magnetization Experimental Data
}

\author{
P. HrubovČÁK ${ }^{a, b, *}$, N. KuČerka ${ }^{b, c}$, A. ZeleñÁKová ${ }^{a}$ And V. ZeleñÁK ${ }^{d}$ \\ ${ }^{a}$ Pavol Jozef Šafárik University in Košice, Department of Condensed Matter Physics, Košice, Slovakia \\ ${ }^{b}$ Joint Institute for Nuclear Research, Frank Laboratory of Neutron Physics, Dubna, Russian Federation \\ ${ }^{c}$ Comenius University in Bratislava, Department of Physical Chemistry of Drugs, Bratislava, Slovakia \\ ${ }^{d}$ Pavol Jozef Šafárik University in Košice, Department of Inorganic Chemistry, Košice, Slovakia \\ Small angle neutron scattering and magnetization data were utilized for revelation of internal structure of \\ advanced nanocomposite containing $\mathrm{Fe}_{2} \mathrm{O}_{3}$ nanoparticles embedded in regular pores of amorphous silica matrix \\ SBA15. Experimental data were analyzed based on appropriate models and the parameters obtained were compared \\ to structural and magnetic characteristics of the system obtained employing other methods. Excellent agreement \\ of the results suggests that mutual application of the methods can provide reliable information about the structures \\ buried in the body of the composite.
}

DOI: 10.12693/APhysPolA.137.730

PACS/topics: neutron scattering, nanocomposite, mesoporous silica, nanoparticles, $\mathrm{Fe}_{2} \mathrm{O}_{3}$

\section{Introduction}

Periodic nanoporous silica SBA15 with its perfect regular structure, biocompatibility, thermal stability and durability, and high specific surface is very promising material from the point of view of application. It can serve as a hosting medium for various structures (magnetic nanoparticles, drugs) resulting in formation of advanced nanocomposite systems. Unique properties of particular subsystems that are combined in the tuned composite enable its potential utilization in biomedicine (targeted drug delivery, hyperthermia, contrast agents [1]) or magnetic refrigeration [2]. Several reliable methods of specific material deposition into matrix pores have been proposed. However, determination of characteristics of nanostructures, that have been created in the body of the matrix, remains a challenge. X-rays, regarded as standard tool of structural analysis, are somewhat limited in examination of the bulk region of highly absorbing materials. In the particular case of amorphous silica, neutrons appear to be more feasible probes providing (along with other complementary methods) plausible information on matter organization deeper in the sample body. This is due to their high penetrability and different contrast between silica and nanoobjects when compared to, for example, X-ray scattering. Small angle neutron scattering (SANS) is the method that is frequently used when scrutinizing structures and magnetic systems at nanoscale [3]. It has been applied for the investigation of ferrofluids [4], magnetoferritin

*corresponding author; e-mail: pavol.hrubovcak@upjs.sk in aqueous solution [5], or the adsorption of magnetite nanoparticles on lysozyme amyloid fibrils [6].

In this study, we investigate the internal structure of nanocomposite consisting of $\mathrm{Fe}_{2} \mathrm{O}_{3}$ nanoparticles (NPs) embedded in amorphous silica matrix by means of SANS and magnetization measurements. We applied appropriate models that are tailored to our particular system in order to fit experimental data and obtain NP size distribution along with other structural characteristics.

\section{Experimental}

Material under examination is nanocomposite that contains magnetic NPs of $\alpha-\mathrm{Fe}_{2} \mathrm{O}_{3}$ hematite phase embedded in the regular longitudinal nanopores (average diameter $\approx 8 \mathrm{~nm})$ of amorphous silica $\left(\mathrm{SiO}_{2}\right)$ matrix. Details of preparation are reported in $[7,8]$. It is produced in powder form with average grain size of about $500 \mathrm{~nm}$. Transmission electron microscope image along with schematic illustration of the system is shown in Fig. 1 (inset), while its profound structural and magnetic characterization is available elsewhere (sample denoted as $\mathrm{Fe}_{2} \mathrm{O}_{3} @ \mathrm{SBA} 15$ 0.5M) [7, 8]. There is ample evidence of superparamagnetic NPs being present in the pores of the hosting matrix, however, information on the particles' shapes and size distribution that is crucial for understanding the magnetic behavior of the whole composite, is absent.

The dependence of magnetization on field magnitude (up to $5 \mathrm{~T}$ ) was recorded at room temperature by means of Quantum Design MPMS 5XL magnetometer on the basis of SQUID. The powder sample in a gelatin capsule was measured and diamagnetic contributions of capsule and plastic sample holder were subtracted from experimental data. 
Small angle neutron scattering experiments were performed at the IBR-2 pulsed reactor, JINR (Dubna, Russia) employing spectrometer YuMO. Powder sample was loaded in alumina cell and exposed to neutrons for one hour at room temperature. The scattering from empty cell was subtracted from the scattering of the sample while data processing.

The model was applied that attempts to describe experimental SANS intensity dependence on momentum transfer $I(q)$ by taking into account all relevant structural features of investigated nanocomposite. General idea stems from a principle of superposition, where total scattering intensity of the sample is assumed to consist of two independent, thus additive contributions - scattering from hosting matrix $\left(I_{M}\right)$ and scattering from $\mathrm{Fe}_{2} \mathrm{O}_{3}$ NPs $\left(I_{\mathrm{NP}}\right)$ [9]. We have extended the existing model for hollow silica $[10,11]$ adding the term $I_{\mathrm{NP}}$ considering neutron scattering from non-interacting polydisperse $\mathrm{Fe}_{2} \mathrm{O}_{3}$ objects. The cross-terms are neglected due to low enough concentration of NPs. At room temperature, the subsystem of NPs is in superparamagnetic state [1], hence mutual magnetic interparticle interactions can be ruled out. In order to scrutinize the possibility of nanoparticle aggregation within the pores, several shapes of nanoobjects (spheres, rods, wires) were assumed and tested in the model. The best results were obtained for spherical polydisperse (Gaussian size distribution) NPs. In this case scattering is proportional to the square of form factors of individual nanoparticle of radius $R$, its volume $V$, and neutron scattering length density $\rho_{\mathrm{NP}}$ embedded in medium with $\rho_{0}[12]$ :

$$
\begin{gathered}
P_{\mathrm{NP}}(q, R)=\frac{C_{1}}{V}\left[\frac{3 V\left(\rho_{\mathrm{NP}}-\rho_{0}\right)}{(q R)^{3}}\right]^{2} \\
\times[\sin (q R)-q R \cos (q R)]^{2}+C_{2},
\end{gathered}
$$

$C_{1}$ being the scaling coefficient depending on forward scattering $I(q=0)$, and $C_{2}$ being the background constant.

Magnetic properties of the nanocomposite were modeled utilizing the Langevin law. The polydispersity of magnetic moments corresponding to NPs of different sizes was taken into account leading the expression for magnetization vs. applied magnetic field dependence of magnetic moments system [13]:

$$
M(B)=\frac{M_{s}}{\left\langle z^{3}\right\rangle} \int_{0}^{\infty} z^{3} L\left(\frac{M_{s b} V_{m} B}{k_{\mathrm{B}} T} z^{3}\right) F_{N}(z) \mathrm{d} z,
$$

where $M_{s}$ and $M_{s b}$ are saturation magnetizations of the nanoparticle system and bulk $\mathrm{Fe}_{2} \mathrm{O}_{3}$ material, respectively, $B$ is applied magnetic field, $k_{\mathrm{B}}$ is the Boltzmann constant, $T$ is temperature, and $V_{m}$ is the particles' median volume. Log-normal number-weighted distribution $F_{N}(z)$ of reduced particle diameters $\left(z=D / D_{m}\right)$ was employed in the model, where $D_{m}$ is median diameter and $\left\langle z^{3}\right\rangle=\int z^{3} F_{N}(z) \mathrm{d} z$ is median reduced particle volume.

\section{Results and discussion}

Application of the model to SANS experimental data is demonstrated in Fig. 1 (red curve). In the scattering contribution of hollow hosting matrix (green curve), one can recognize diffraction peaks at $q=0.07 \AA^{-1}$ and $q=0.12 \AA^{-1}$, that correspond to scattering of cylindrical nanopores of radius $R_{p}=4 \mathrm{~nm}$ (standard deviation $\sigma_{p}=0.5 \mathrm{~nm}$ ) that are ordered in $2 \mathrm{D}$ hexagonal lattice with pore center-to-center distance $a_{0}=10 \mathrm{~nm}$ $\left(\sigma_{a 0}=0.58 \mathrm{pm}\right)$. Since HRTEM micrographs of the system provided the evidence of larger NPs occurrence at the surface of the nanocomposite [8], two systems of $\mathrm{Fe}_{2} \mathrm{O}_{3}$ NPs were assumed to contribute to total scattering. Adjusting fitting parameters and the shape of $\mathrm{Fe}_{2} \mathrm{O}_{3}$ objects allowed to establish spherical rather than rod-like or nanowire-like shape. According to the model, nanoparticles of average radius $R_{M}=3.5 \mathrm{~nm}$ $\left(\sigma_{R M}=0.7 \mathrm{~nm}\right)$ occupied the pores of the matrix (Fig. 1, blue curve) and a small fraction of NPs of average radius $R_{S}=10 \mathrm{~nm}\left(\sigma_{R S}=3.8 \mathrm{~nm}\right)$ were spread on the nanocomposite surface (Fig. 1, yellow curve).

In order to corroborate the particle size distribution, system's magnetization dependence on applied field magnitude $M(H)$ was modeled and fitted to the experimental data. Despite antiferromagnetic nature of bulk $\alpha-\mathrm{Fe}_{2} \mathrm{O}_{3}$ phase, the examined nanocomposite exhibits clear signatures of superparamagnetic behavior [7, 8]. The reason is twofold. At temperatures above $T_{M} \approx 263 \mathrm{~K}$ (the Morion temperature), the moments of atoms lie in the basal (111) plane of crystal lattice with a slight canting away from the antiferromagnetic axis, resulting in a small net magnetization. This effect is enhanced in

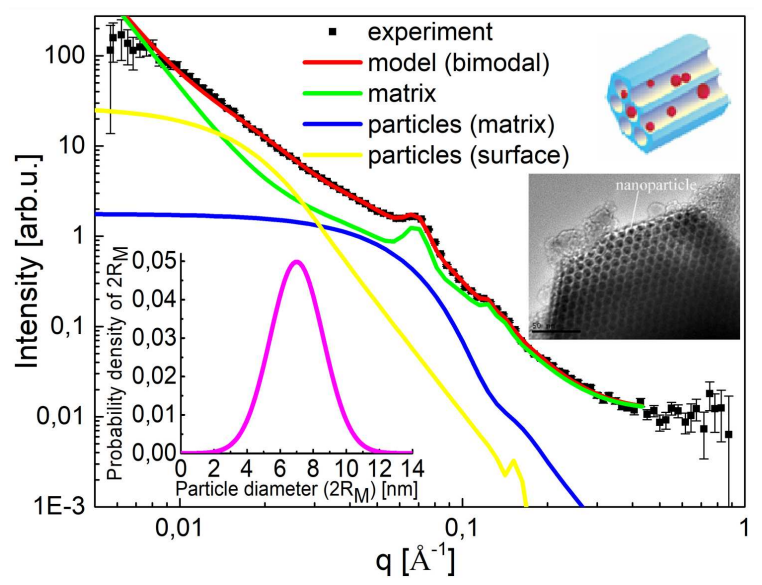

Fig. 1. The model fit (red curve) to SANS experimental data (black squares) decomposed to contributions from hollow silica matrix (green curve) and system of $\mathrm{Fe}_{2} \mathrm{O}_{3}$ nanoparticles embedded in the matrix pores (blue curve) and spread on the surface of nanocomposite (yellow curve). Insets show TEM image and scheme of the composite (right hand side), and size distribution of nanoparticles embedded in matrix determined from the fit (left hand side). 


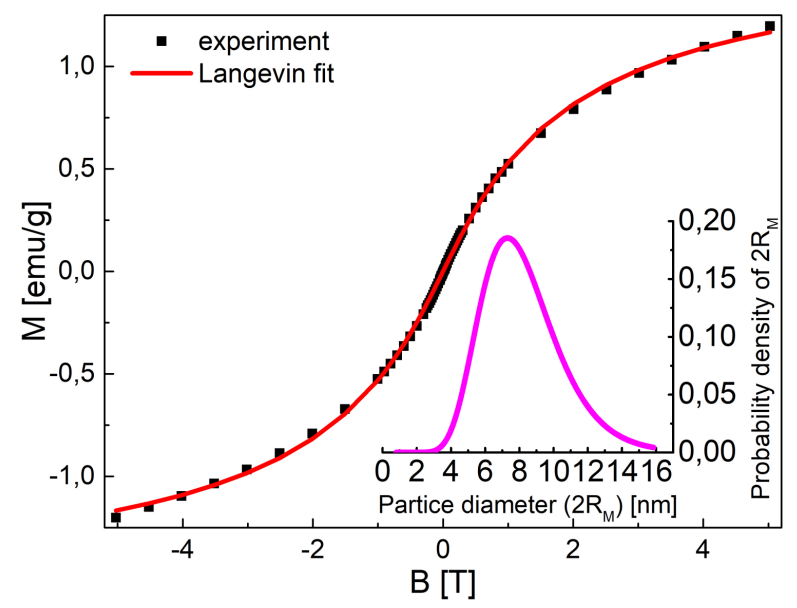

Fig. 2. The dependence of magnetization on the applied magnetic field magnitude in the case of examined nanocomposite measured at $T=300 \mathrm{~K}$. Red curve represents fit of Eq. (2) to the experimental data. Inset shows the nanoparticle size distribution established in the fitting.

the case of fine nanoparticles [14]. Further, and more importantly, due to high surface-to-volume ratio, uncompensated spins from surface layers contribute significantly to the net magnetization of particles. Relatively large $M_{s}$ values of $4-10 \mathrm{emu} / \mathrm{g}$ reported for fine (2-10 nm) hematite NPs were ascribed to this phenomenon [15, 16]. Since the model behind Eq. (2) was proposed for ferromagnetic materials and does not assume vast difference between $M_{s}$ and $M_{s b}$ expected in our system, we identified saturation magnetization of bulk $\alpha-\mathrm{Fe}_{2} \mathrm{O}_{3}\left(M_{s b}=0.4 \mathrm{emu} / \mathrm{g}\right.$ [17] $)$ with the value of $M_{s}=1.5 \mathrm{emu} / \mathrm{g}$ estimated from experimental $M(H)$ data (Fig. 2). The best fit of Eq. (2) to the $M(H)$ data recorded at temperature $300 \mathrm{~K}$ is shown in Fig. 2. Log-normal distribution of particle diameters was established with median diameter $7.9 \mathrm{~nm}\left(\sigma_{\text {mag }}=1.32 \mathrm{~nm}\right)$ that corresponds to individual nanoparticle moment $m_{p}=229 \mu_{\mathrm{B}}\left(\mu_{\mathrm{B}}\right.$ is Bohr magneton $)$ with saturation magnetization $1.55 \mathrm{emu} / \mathrm{g}$. Intriguingly, results from magnetic analysis are consistent with the findings derived from SANS experiments. Moreover, they reinforce the conclusions on the average nanoparticle size and magnetic moment in the examined nanocomposite from earlier studies $[7,8]$.

\section{Conclusions}

As it has been demonstrated, SANS dependence on momentum transfer can be modeled providing structural characteristics of the subsystems of examined $\mathrm{Fe}_{2} \mathrm{O}_{3} @$ SBA15 nanocomposite. In this study, the size distribution of $\mathrm{Fe}_{2} \mathrm{O}_{3}$ NPs occupying mesopores of SBA15 matrix was determined both from SANS and magnetization data. Model-based data analysis provided the results that are in fairly good accordance also with the results obtained by other experimental methods. This shows that models applied can be regarded as reliable tools for the examination of SBA15 nanocomposite inner structure.

\section{Acknowledgments}

This work was supported by the Slovak Research and Development Agency under the contracts APVV-15-0520, and by the VEGA projects No. $1 / 0745 / 17$, No. $1 / 0143 / 20$.

\section{References}

[1] O. Kapusta, A. Zeleňáková, P. Hrubovčák, V. Girman, V. Zeleňák, Acta Phys. Pol. A 131, 860 (2017).

[2] A. Zeleňáková, P. Hrubovčák, O. Kapusta, V. Zeleňák, V. Franco, Appl. Phys. Lett. 109 (2016).

[3] S. Mühlbauer, D. Honecker, É.A. Périgo, et al., Rev. Mod. Phys. 91, 015004 (2019).

[4] M.V. Avdeev, V.I. Petrenko, A.V. Feoktystov, I.V. Gapon, V.L. Aksenov, L. Vékás, P. Kopčanský, Ukr. J. Phys. 60, 728 (2015).

[5] L. Melníková, V.I. Petrenko, M.V. Avdeev, V.M. Garamus, L. Almásy, O.I. Ivankov, L.A. Bulavin, Z. Mitróová, P. Kopčanský, Coll. Surf. B 123, 82 (2014).

[6] J. Majorošová, V.I. Petrenko, K. Šipošová, et al., Coll. Surf. B 146, 794 (2016).

[7] A. Zeleňáková, V. Zeleňák, J. Bednarčík, P. Hrubovčák, J. Kováč, J. Alloys Comp. 582, 483 (2014).

[8] V. Zeleňák, A. Zeleňáková, O. Kapusta, P. Hrubovčák, V. Girman, J.B ednarčík, RSC Adv. 9, 3679 (2019).

[9] A. Zeleňáková, P. Hrubovčák, O. Kapusta, N. Kučerka, A. Kuklin, O. Ivankov, V. Zeleňák, Sci. Rep. 9, 15852 (2019).

[10] T. Shin, G.H. Findenegg, A. Brandt, Prog. Coll. Polym. Sci. 133, 116 (2006).

[11] M. Imperor-Clerc, P. Davidson, A. Davidson, J. Am. Chem. Soc. 122, 11925 (2000).

[12] SasView 5.0.2 documentation, Sphere Functions, 2019.

[13] M. El-Hilo, J. Appl. Phys. 112, (2012).

[14] F. Bodker, M.F. Hansen, C.B. Koch, K. Lefmann, S. Morup, Phys. Rev. B 61, 6826 (2000).

[15] J.L. Dormann, J.R. Cui, C. Sella, J. Appl. Phys. 57, 4283 (1985).

[16] M. Tadić, D. Marković, V. Spasojević, V. Kusigerski, M. Remškar, J. Pirnat, Z. Jagličić, J. Alloys Comp. 441, 291 (2007)

[17] M. Ahmadzadeh, C. Romero, J. McCloy, AIP Adv. 8, 056807 (2018). 